\title{
Scrub Typhus - A Major Cause of Pediatric Intensive Care Admission and Multiple Organ Dysfunction Syndrome: A Single-Center Experience from India
}

\author{
Prabahs Prasun Giri, Joydeb Roy ${ }^{1}$, Agnisekhar Saha \\ Pediatric Intensive Care unit, Institute of Child Health, 'Department of Pediatrics, Institute of Child Health, Kolkata, West Bengal, India
}

Abstract

\begin{abstract}
Aim of the Study: Scrub typhus has been globally recognized as an emerging infectious disease contributing significantly to pyrexia of unknown origin (PUO) and a potential cause of multiorgan dysfunction syndrome (MODS). We studied the incidence of scrub typhus as a cause of pediatric intensive care unit (PICU) admission and MODS in our hospital and its clinical and laboratory characteristics to measure the incidence of MODS caused by scrub typhus. Materials and Methods: This study was done in a pediatric teaching hospital in Kolkata, India. Records of patients admitted with PUO from March 2012 to December 2015 were reviewed. Rathi-Goodman-Aghai scoring system was used to identify potential ST patients and confirmed by serological testing. Clinical characteristics, laboratory findings, and treatment response were noted of those needing PICU admissions. Results: Ninety-seven cases of scrub typhus have been identified during that period. PICU admission was needed in 30 of them (31\%) which contributed 8.43\% of total PICU admissions. Among these 30 patients, 16 (53\%) developed MODS which contributed $18.29 \%$ of total MODS admitted in PICU. Septic shock was the most common manifestation in as many as $18(60 \%)$ patients followed by encephalopathy in $13(43 \%)$. Patients were treated with either doxycycline alone or in combination with azithromycin. Mean time to complete defervescence was $32 \mathrm{~h}$ after first dose of doxycycline. The outcome was excellent without a single mortality. Conclusion: Scrub typhus is an important cause of MODS in this part of the world, especially in fevers associated with features as identified and not responding to conventional antibiotics.
\end{abstract}

Keywords: Fever, multiorgan dysfunction syndrome, scrub typhus

\section{INTRODUCTION}

Rickettsial diseases are some of the most covert reemerging infections of the present times. They are generally incapacitating and notoriously difficult to diagnose as their presentation resembles that of viral, bacterial, or protozoal diseases. This may lead to unnecessary investigation and treatment causing considerable morbidity, irreversible damage to vital organ, and finally even death. Untreated cases can have fatality rates as high as $30 \%-35 \%$, but when diagnosed properly, they are often easily treated. ${ }^{[1]}$ Scrub typhus, the most common of the Rickettsial diseases in India, is being increasingly reported in our country for the last couple of years. ${ }^{[2]}$ It is highly endemic in suburban region. It is an acute febrile illness caused by Orientia tsutsugamushi and is transmitted by bite of larval stage of trombiculid mite or chigger. ${ }^{[3]}$ The classical symptomatic presentation of the disease is the so-called spotted fever

\begin{tabular}{|l|l|}
\hline \multicolumn{2}{|c|}{ Access this article online } \\
\hline Quick Response Code: & Website: \\
\hline & www.ijccm.org \\
\hline & \\
\hline
\end{tabular}

which typically evolves from an initial nonspecific clinical picture of fever, headache, and myalgia to the development of a purpuric palpable rash starting from the extremities with or without an eschar. However, this is rarely seen in pediatric population and that makes the diagnosis difficult at times. ${ }^{[4]}$ However, the positive aspect of this condition lies in the fact that they can easily be diagnosed by cheap, inexpensive blood test (Weil Felix test and IgM ELSIA) and can be treated with less expensive oral drugs such as doxycycline safely even in the pediatric population of below 8 years of age if high

Address for correspondence: Dr. Prabhas Prasun Giri, Flat C1, Anandan, 173, Sarat Ghosh Garden Road, Dhakuria, Kolkata - 700 031, West Bengal, India. E-mail: dr.prabhas@yahoo.co.in

This is an open access article distributed under the terms of the Creative Commons Attribution-NonCommercial-ShareAlike 3.0 License, which allows others to remix, tweak, and build upon the work non-commercially, as long as the author is credited and the new creations are licensed under the identical terms.

For reprints contact: reprints@medknow.com

How to cite this article: Giri PP, Roy J, Saha A. Scrub typhus - A major cause of pediatric intensive care admission and multiple organ dysfunction syndrome: A single-center experience from India. Indian J Crit Care Med 2018;22:107-10. 
degree of suspicion is maintained. Here, we present our study about the incidence of ST as a cause of pediatric intensive care unit (PICU) admission and multiorgan dysfunction syndrome (MODS) in our hospital and its clinical and laboratory characteristics in the time frame of last $3 \frac{1}{2}$ years.

\section{Materials and Methods}

Clinical records of children aged below 15 years admitted in a pediatric teaching hospital in Kolkata, with pyrexia of unknown origin (PUO) during the time period of March 2012 to December 2015 were reviewed. Rathi-Goodman-Aghai scoring system ${ }^{[5]}$ was used to identify potential ST patients and confirmed by serological testing, i.e., IgM ELISA for scrub typhus. Serologically confirmed scrub typhus patients needing PICU admission were identified and included in the study. The data collected included details of travel history, clinical and laboratory features, treatment, and outcome. MODS was defined as simultaneous involvement of more than 2 organ systems. All the patients were treated with IV doxycycline $(5 \mathrm{mg} / \mathrm{kg} /$ day $)$ followed by oral doxycycline for a total of 7-10 days depending on the clinical response and severity of the disease. The time of defervescence and return of normal laboratory parameters were also noted. Appropriate ethical approval was obtained from the Ethical Committee of the hospital, Kolkata.

\section{RESULTS}

\section{Clinical features}

During the study period, a total of 861 cases of PUO were admitted in the pediatric ward. ST was the serologically confirmed final diagnosis in 97 out of that 861 children, i.e., $11 \%$ of PUO admissions. PICU admission was needed in 30 of them (31\%) which contributed $8.43 \%$ of total PICU admissions.

Among these 30 patients, 16 (53\%) developed MODS which contributed $18.29 \%$ of total MODS admitted in PICU. Septic shock was the most common manifestation in as many as $18(60 \%)$ patients followed by encephalopathy in $13(43 \%)$. Acute respiratory distress syndrome (ARDS)/acute lung injury was present in 4 patients at presentation and later on developed in another six patients as a manifestation of shock. Acute liver injury, acute kidney injury (AKI), and coagulopathy was present in, respectively, 11, 9, and 7 patients. Beside these, 25 of them either had a history of rash or had a rash during hospital admission and 21 had generalized lymphadenopathy. Some other clinical features of these 30 patients are given in Table 1 .

\section{Laboratory features}

Scrub typhus IgM ELISA was the test done to confirm the diagnosis in all the cases. We also did Well-Felix test, and it came out to be positive in 25 patients (83\%). OXK $>1: 160$ was taken as positive. C-reactive process was universally raised in all. Hyponatremia and hypoalbuminemia were also quite common. Few of the cases also demonstrated cytopenias

\begin{tabular}{lcc}
\hline \multicolumn{3}{l}{ Table 1: Clinical features of patients } \\
\hline Clinical features & $\begin{array}{r}\text { Total number of } \\
\text { patients }(\boldsymbol{n = 3 0 )}\end{array}$ & Percentage \\
\hline Hepatomegaly & 26 & 87 \\
Splenomegaly & 21 & 70 \\
Rash & 18 & 60 \\
Encephalopathy & 13 & 43 \\
Pedal edema & 13 & 43 \\
Generalized lymphadenopathy & 12 & 40 \\
Respiratory distress & 12 & 40 \\
Conjunctival congestion & 10 & 25 \\
Anasarca & 7 & 23 \\
Eschar & 6 & 20 \\
\hline
\end{tabular}

and altered coagulation status. Laboratory features of these 30 patients are shown in Table 2.

\section{Treatment and outcome}

All the patients were treated with IV doxycycline at a dose of $5 \mathrm{mg} / \mathrm{kg} /$ day in a once-daily dose. IV doxycycline was converted to oral doxycycline capsules when they were able to swallow capsule. In $60 \%$ cases, fever subsided after single dose of doxycycline, and almost all of them became afebrile after $72 \mathrm{~h}$ of starting doxycycline. The mean duration of complete defervescence was $32 \mathrm{~h}$. Seven patients needed mechanical ventilation and 5 needed blood products' transfusion. The average duration of PICU stay was 5.2 days. Three of them had secondary nosocomial infection that substantially increased the PICU stay. Twenty-seven out of 30 came for follow-up and all of them did well in the $2^{\text {nd }}$ week follow-up.

\section{Discussion}

Multiple organ dysfunction syndrome (MODS) is the presence of altered organ function in acutely ill patients such that homeostasis cannot be maintained without intervention. It usually involves two or more organ systems. ${ }^{[6]}$ MODS is the most common cause of ICU admission and mortality in both adult and pediatric population and the most common underlying cause of MODS is sepsis in both the developed and developing countries. ${ }^{[7]}$ In our institute, ST is found to be one of the major causes of MODS and PICU admission. However, unlike septic shock, the early diagnosis and timely intervention usually lead to an excellent outcome as in our cohort the survival rate was $100 \%$.

Scrub typhus is an acute febrile illness, and it should be considered in the differential diagnosis of patients with acute febrile illness, including those with altered sensorium, pneumonitis, atypical pneumonia, ARDS, thrombocytopenia, and abnormalities in liver function tests. ${ }^{[8]}$ The spectrum of disease ranges from fever, sore throat, cough, myalgia, headache, rash, and the formation of an eschar to life-threatening encephalitis, interstitial pneumonia, myocarditis, pericarditis, cardiac arrhythmia, acute renal failure, acute liver failure, and ARDS. ${ }^{[9]}$ In recent times, Rickettsial disease has been an important cause of reemerging infection all over the 


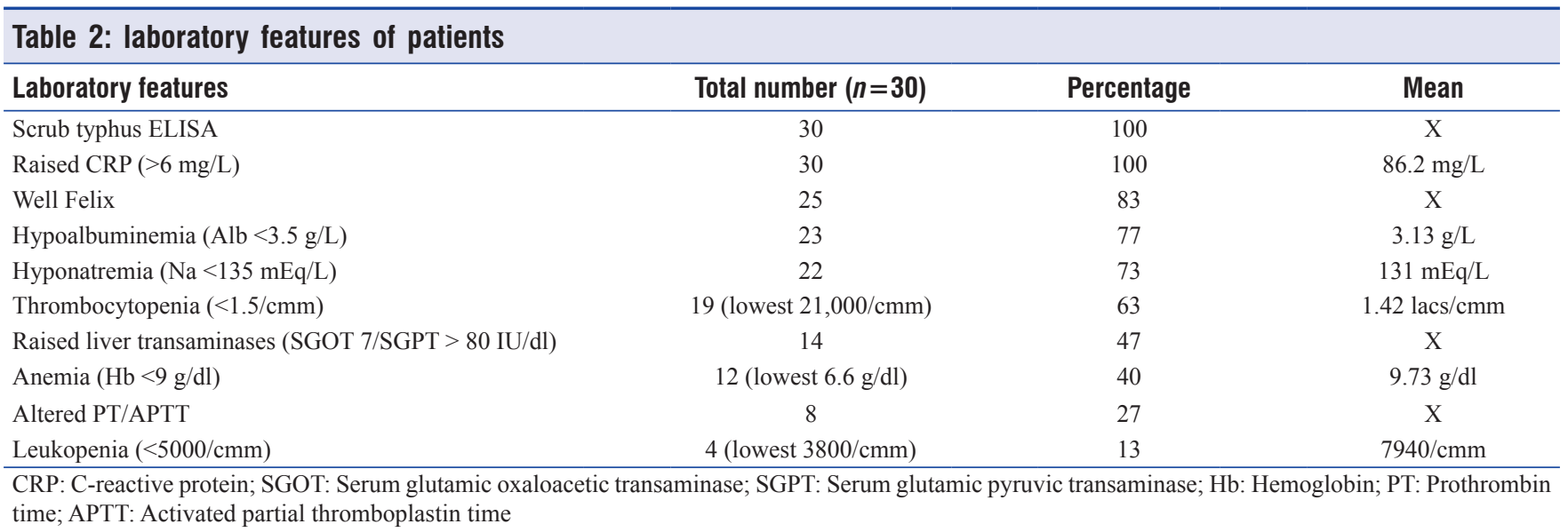

world and ST is the most common in India in both adult and pediatric populations. Across the globe, more than one billion people live in endemic areas and are exposed to the risk of the disease. India has an annual incidence of approximately one million cases. ${ }^{[10]}$ The disease was first reported in army barracks during World War II in Assam and West Bengal, again during Indo- Pakistani war in 1965. Interest was renewed with outbreaks in the sub-Himalayan belt from Jammu to Nagaland and Haryana ${ }^{[11,12]}$ and in 2012 from West Bengal. It is also being increasingly recognized as a potential cause of organ dysfunction as well as Intensive Care Unit admission in all age group. There are plenty of case reports and case series in adults about ST and MODS. ${ }^{[13-16]}$ However, the data in pediatric population are scanty.

Gurunathan et al. ${ }^{[17]}$ reported the clinical profile of ST in 130 pediatric patients in this study; incidence of bleeding manifestation, altered level of consciousness, decreased urine output (AKI), seizures (manifestation of febrile seizure and meningoencephalitis), and visual disturbances were $18.5 \%$, $18.5 \%, 10.8 \%, 10 \%$, and $6.9 \%$, respectively, and overall mortality was $6.2 \%$. However, in other studies by Palanivel et al. ${ }^{[18]}$ and Chrispal et al. ${ }^{[19]}$ the mortality was higher $11.94 \%$ and $12.2 \%$, respectively.

In another series from Jaipur, India, Meena et al. ${ }^{[20]}$ presented 49 cases of scrub typhus among which $14.3 \%$ had meningitis and encephalopathy where ARDS, AKI, and shock was present in, respectively, $5.7 \%, 3.8 \%$, and $3.8 \%$ of patients. About $20 \%$ of patient needed PICU or HDU care, $8 \%$ patients had more than 2 organ system involvements, and 4\% patients expired.

Ratageri et al..$^{[21]}$ presented the clinical profile of 36 children of scrub typhus from South India where the rate of complications and MODS was bit lower. Severe hepatitis, CNS involvement, and extensive gangrene and necrosis were seen in one of the each cases.

Although the number of ICU admissions and multiorgan dysfunction is more in our series, the mortality is nil possibly because of early implementation of empiric doxycycline therapy and good PICU care.

\section{Conclusion}

Scrub typhus is an important cause of MODS in this part of the world, especially in fevers associated with features as identifi ed and not responding to conventional antibiotics.

\section{Financial support and sponsorship}

Nil.

\section{Conflicts of interest}

There are no conflicts of interest.

\section{RefEREnCes}

1. Batra HV. Spotted fevers and typhus fever in Tamil Nadu - Commentary. Indian J Med Res 2007;126:101-3.

2. Rathi N, Rathi A. Rickettsial infections: Indian perspective. Indian Pediatr 2010;47:157-64.

3. Jayaram Paniker CK. Ananthanarayan and Paniker's Textbook of Microbiology. $7^{\text {th }}$ ed. University Press Pvt. Ltd.; 2008. p. 412-21.

4. Pavithran S, Mathai E, Moses PD. Scrub typhus. Indian Pediatr 2004:41:1254-7.

5. Rathi NB, Rathi AN, Goodman MH, Aghai ZH. Rickettsial diseases in central India: Proposed clinical scoring system for early detection of spotted fever. Indian Pediatr 2011;48:867-72.

6. Goldstein B, Giroir B, Randolph A; International Consensus Conference on Pediatric Sepsis. International pediatric sepsis consensus conference: Definitions for sepsis and organ dysfunction in pediatrics. Pediatr Crit Care Med 2005;6:2-8.

7. Marshall JC, Cook DJ, Christou NV, Bernard GR, Sprung CL, Sibbald WJ, et al. Multiple organ dysfunction score: A reliable descriptor of a complex clinical outcome. Crit Care Med 1995;23:1638-52.

8. Rungta N. Scrub typhus: Emerging cause of multiorgan dysfunction. Indian J Crit Care Med 2014;18:489-91.

9. Saxena A, Khiangte B, Tiewsoh I. Scrub typhus complicated by acute respiratory distress syndrome and multiorgan failure; an unrecognized alarming entity in central india: A report of two cases. J Family Med Prim Care 2014;3:80-3.

10. Kumar P, Subanna AI, Dharinitragada S, Suri K. Multi-organ dysfunction in scrub typhus. Ann Trop Med Public Health 2012;5:393-6.

11. Koh GC, Maude RJ, Paris DH, Newton PN, Blacksell SD. Diagnosis of scrub typhus. Am J Trop Med Hyg 2010;82:368-70.

12. Varghese GM, Trowbridge P, Janardhanan J, Thomas K, Peter JV, Mathews $\mathrm{P}$, et al. Clinical profile and improving mortality trend of scrub typhus in South India. Int J Infect Dis 2014;23:39-43.

13. Griffith M, Peter JV, Karthik G, Ramakrishna K, Prakash JA, Kalki RC, et al. Profile of organ dysfunction and predictors of mortality in severe scrub typhus infection requiring intensive care admission. Indian J Crit Care Med 2014;18:497-502. 
14. Lee CS, Hwang JH, Lee HB, Kwon KS. Risk factors leading to fatal outcome in scrub typhus patients. Am J Trop Med Hyg 2009;81:484-8

15. Loomba V, Mani A, John M, Oberoi A. Scrub typhus in Punjab: An acute febrile illness with multisystem involvement. Trop Doct 2014;44:152-5.

16. Lee WS, Wang FD, Wang LS, Wong WW, Young D, Fung CP, et al. Scrub typhus complicating acute respiratory distress syndrome: A report of two cases. Zhonghua Yi Xue Za Zhi (Taipei) 1995;56:205-10.

17. Gurunathan PS, Ravichandran T, Stalin S, Prabu V, Anandan H. Clinical profile, morbidity pattern and outcome of children with scrub typhus. Int J Sci Stud 2016;4:247-50.

18. Palanivel S, Nedunchelian K, Poovazhagi V, Raghunadan R,
Ramachandran P. Clinical profile of scrub typhus in children. Indian J Pediatr 2012;79:1459-62.

19. Chrispal A, Boorugu H, Gopinath KG, Prakash JA, Chandy S, Abraham OC, et al. Scrub typhus: An unrecognized threat in South India - Clinical profile and predictors of mortality. Trop Doct 2010;40:129-33.

20. Meena JK, Khandelwal S, Gupta P, Sharma BS. Scrub typhus meningitis: An emerging infectious threat. IOSR J Dent Med Sci 2015; 14:26-32.

21. Ratageri HV, Madhu PK, Sindhu MV, Illalu S, Shepur TA. Clinico-laboratory profile and outcome of rickettsia in children: Hubli (Karnataka) experience. Pediatr Infect Dis 2014;6:3-6. 